\title{
Relación de la composición corporal, las cualidades físicas y funciones cognitivas en estudiantes de educaciónfísica \\ Relation between body composition, physical qualities, and cognitive function in students of physical education \\ *Fernando Maureira Cid, *Patricia Bravo Rojas, *Nicolas Aguilera Godoy, **Valentina Bahamondes Acevedo, ***Carlos Véliz Veliz \\ *Universidad Católica Silva Henríquez (Chile), ** Universidad Metropolitana de Ciencias de la Educación (Chile), *** Gimnasio Pulse, Estadio Mayor (Chile)
}

\begin{abstract}
Resumen. Existen resultados controversiales sobre la relación de la antropometría, las cualidades físicas y el rendimiento en pruebas cognitivas. En tanto, algunos trabajos muestran relación entre el IMC, el porcentaje de grasa o la capacidad aeróbica con la atención, memoria o control inhibitorio, otros no muestran relaciones significativas. El objetivo del presente estudio fue relacionar la composición corporal, las cualidades físicas, la inteligencia, la atención y la memoria en una muestra constituida por 125 estudiantes que cursan la carrera de pedagogía en educación física. Al total de la muestra se le evalúo las cualidades físicas y funciones cognitivas y a 50 estudiantes se les realizó el estudio antropométrico. Los resultados no muestran diferencias en los resultados de las funciones cognitivas según sexo, pero los varones presentan mayores índices en las cualidades físicas, en masa muscular y en diámetros antropométricos, y las damas presentan mayores índices en pliegues cutáneos. También se encontraron relaciones bajas entre la potencia de brazos y piernas con la inteligencia y la atención sostenida, en tanto, la memoria y la atención selectiva no presentaron relación con ninguna de las variables físicas evaluadas. Estos resultados son similares a otras investigaciones antropométricas y de cualidades físicas en estudiantes de educación física. La relación con variables cognitivas es coherente con trabajos anteriores, asumiendo que una mejor condición física permite una mejor irrigación sanguínea, aumento de la vascularización, neurogénesis y sinaptogénesis, lo cual puede influenciar en la mejora en pruebas cognitivas. Palabras claves: antropometría, cognición, fuerza, capacidad aeróbica, educación física.
\end{abstract}

Abstract. Controversial results are shown on the relation between anthropometry, physical qualities, and performance in cognitive tests. While some works pinpoint the relation of BMI, percentage of fat, or aerobic capacity with attention, memory, or inhibitory control, others do not find significant relations. The aim of the present study was to relate body composition, physical qualities, intelligence, attention, and memory. The sample was constituted by 125 students enrolled in the undergraduate program of pedagogy in physical education. Physical qualities and cognitive functions were assessed in the whole sample, whereas 50 students also carried out anthropometric evaluations. No differences were found in cognitive function by gender; however, males presented higher indexes in physical qualities, in muscular mass, and in anthropometric diameters, whereas females presented higher indexes in cutaneous folds. Also, low relations were found between arm and leg power and intelligence and supported attention, whilst memory and selective attention did not show any relation with any of the physical variables evaluated. Our outcomes are similar to those from previous research on anthropometry and physical qualities in students of physical education. The relation with cognitive variables is coherent with previous works, assuming that a better physical condition allows a better blood irrigation, increase of the vascularization, neurogenesis and synaptogenesis, which can determine an improvement in cognitive test performance.

Keywords: anthropometry, cognition, force, aerobic capacity, physical education.

\section{Introducción}

La composición corporal y las cualidades físicas has sido relacionadas con diversas funciones cognitivas (Maureira, 2018). Un estudio de Killane, Donoghue, Savva, Cronin, Kenny y Reilly (2014) muestra que una mejor capacidad aeróbica se condice con menores tiempos de reacción y mayor modulación de ondas cerebrales durante la resolución de pruebas de atención visuo-espacial en adultos jóvenes. También se ha observado una relación entre atención sostenida, velocidad de procesamiento y memoria de trabajo con la velocidad de marcha en adultos mayores (Pérez, Padilla, Parmentier \& Andrés, 2014), relación entre la capacidad aeróbica y la atención en escolares (Guillamón, García \& Carrillo, 2019), una asociación entre mayor tiempo semanal de práctica de actividad física y los resultados en test de atención en sujetos adultos jóvenes (Wang, Liang, Tseng, Muggleton, Juan \& Tsai, 2015) y mejora de la atención-concentración con la práctica de actividad en adultos mayores (Gerard, Salicetti, Moncada y Solano, 2018). Una investigación de Hirota, Watanabe, Sun, Tanimoto, Kono, Takasaki, et al. (2010) reveló que bajos niveles de rendimiento físico se relacionaban con bajos niveles de flexibilidad cognitiva en adultos mayores, en tanto, Van der Niet, Smith, Scherder, Oosterlaan, Hartman y Visscher (2015) mostraron que altos niveles de sedentarismo se relacionaban negativamente con el control de impulsos en niños de entre ocho y 12 años. Otros estudios describen que altos niveles de IMC y porcentaje de grasa se

Fecha recepción: 16-09-18. Fecha de aceptación: 16-01-19 Fernando Maureira Cid maureirafernando@yahoo.es constituían como indicadores de bajo control inhibitorio en adolescentes (Huang, Tarp, Domazet, Thorsen, Froberg, Andersen, et al., 2015) y que la obesidad se asocia con una disminución en la memoria, funciones ejecutivas y velocidad de procesamiento (Deckers, Van Boxtel, Verhey \& Köhler, 2017).

Pese a lo anterior, existen estudios que no revelan relaciones positivas entre antropometría y cognición. Una investigación en mujeres premenopaúsicas sanas muestra independencia entre la obesidad y la precisión en tareas cognitivas de atención, memoria y planificación, aunque muestra una relación negativa entre obesidad mórbida y longitud de memoria (Farooq, Gibson, Reilly \& Gaoua, 2018), incluso en adultos mayores se ha observado la paradoja de la obesidad donde se describe una asociación entre obesidad y resultados favorables de salud (Braun, Gomes y Schütz, 2015) incluidas funciones cognitivas como la atención y flexibilidad mental (Skinner, Abel, McCoy \& Wilkins, 2017).

En este escenario controversial se hace necesario el estudio de las relaciones entre composición corporal y funciones cognitivas en diversas poblaciones, ya que parece ser que la edad, sexo, raza y algunas patologías (Cohen, Signorello, Cope, McLaughlin, Hargreaves, Zheng, et al., 2012; Eichen, Matheson, Liang, Strong, Rhee \& Boutelle, 2018; Goel, Lopez-Jiménez, De Schutter, Coutinho \& Lavie, 2014; Mullor, Gallego, Cangas, Aguilar-Parra, Valenzuela, Mateu, et al., 2017) podrían influenciar en estas asociaciones. Por lo tanto, el objetivo de este estudio fue examinar la relación entre la composición corporal, cualidades físicas, inteligencia, memoria y atención en estudiantes de Educación Física. Además, se estudia si estas asociaciones son influenciadas por el sexo de la muestra. 


\section{Material y método}

\section{Tipo de estudio}

La presente investigación es de tipo correlacional, ya que busca establecer si existen relaciones entre la composición corporal, las cualidades físicas y funciones cognitivas. El estudio tiene un diseño no experimental, ya que no existió manipulación de las variables y es de corte transversal porque se realizaron las mediciones en un solo momento.

\section{Muestra}

Se trabajó con una muestra no probabilística intencional que estuvo constituida por 125 estudiantes de primer año de la carrera de Pedagogía en Educación Física de una Universidad de Santiago de Chile, que correspondeal 93\% de la población. La edad mínima fue de 18 y la máxima de 23 años, con una media de 18,6 $\pm 1,15$. Del total, 85 estudiantes son de sexo masculino (68\%) y 40 de sexo femenino (32\%). Las mediciones se realizaron como parte de una evaluación de entrada de los estudiantes a la carrera. Para el estudio antropométrico se evaluaron a 50 estudiantes al azar dentro de la muestra total, el tipo de muestreo fue aleatorio simple. Fueron excluidos todos los estudiantes que en el momento de la evaluación estuviesen en recuperación de alguna lesión, que presentaran alguna patología o trastorno de atención o memoria, algún problema nutricional o que estuviesen en tratamiento neurofarmacológico. Esta investigación fue aprobada por el comité de ética de la Universidad Católica Silva Henríquez mediante el acta s/n de marzo de 2018. Todos los participantes firmaron un consentimiento informado.

\section{Instrumentos}

Mediciones antropométricas: se utilizó una balanza mecánica SECA 700 con un tallímetro telescópico SECA 220, con precisión de 50 gramos y un centímetro respectivamente. Para la evaluación de diámetros, perímetros y pliegues cutáneos se utilizó el kit antropométrico ROSSCRAFT. Los datos recolectados fueron peso bruto (kg), talla corporal (cm), talla sentado (cm), diámetros biacromial, tórax transverso, tórax antero-posterior, bi-iliocrestídeo humeral (biepicondilar) y femoral (biepicondilar), perímetros de cabeza, brazo relajado, brazo flexionado en tensión, antebrazo máximo, tórax mesoesternal, cintura mínima, cadera máxima, muslo máximo, muslo medial y pantorrilla máxima, y pliegues cutáneos del tríceps, subescapular, supraespinal, abdominal, muslo medial y pantorrilla máxima (se utilizó un cáliper con precisión de $0,2 \mathrm{~mm}$ y con prensión de cierre de $10 \mathrm{~g} / \mathrm{mm}^{2}$ ). Con estos valores se calculó la masa adiposa, masa muscular, masa residual, masa ósea, piel eíndice músculo-óseo.

Cualidades físicas: se utilizaron las pruebas de abdominales en 60 segundos para evaluar la potencia abdominal, flexo-extensiones de codos en 30 segundos para evaluar la potencia muscular del tren superior, salto largo a pies juntos para evaluar la potencia muscular del tren inferior y el test course Navette para evaluar la capacidad aeróbica. Todas las pruebas son parte de la batería SIMCE (Sistema de Medición dela Calidad dela Educación) de educación física que utiliza el Gobierno de Chile para conocer la condición física de los estudiantes (Agencia de la Calidad en Educación, 2015).

Atención: se utilizó el Test de Toulouse-Pierón, que es una prueba perceptiva creada por Eduardo Toulouse en 1904, considerada como una de las técnicas más relevantes para evaluar la atención (León-Carrión, 1995). La prueba consta de una matriz de 40 filas x 30 columnas de signos (1.200 en total). En la parte superior de la hoja se muestran dos figuras modelos. La finalidad del test es encontrar las figuras que presentan esas características entre las 1.200 que se encuentran en la hoja. La aplicación de la prueba fue realizada en forma colectiva y su duración es de 10 minutos. Esta prueba presenta índices de confiabilidad y validez adecuados en estudiantes de Pedagogía en Educación Física Chilena (Maureira, Trujillo \& Flores, 2014).

Memoria: se utilizó el Test de Retención Visual de Benton forma D creado por Arthur Benton en 1945 (Benton, 1981). Es un instrumento clínico y de investigación, utilizado para evaluar la percepción visual, la memoria visual y las habilidades viso-constructivas. El test consta de 10 láminas constituidas por figuras geométricas de borde negro sobre un fondo blanco. Cada lámina se presenta por 10 segundos tras los cuales deben transcurrir 15 segundos para que el sujeto evaluado comience a dibujar la figura recordada, teniendo 10 segundos para hacerlo. La valoración del test se realiza en base a los aciertos y errores en cada una de las láminas dibujadas. Los errores están dados por omisiones de figuras, distorsiones, perseverancias, rotaciones, desplazamientos y errores de tamaño. Este test presenta índices de confiabilidad y validez adecuados en estudiantes de Pedagogía en Educación Física Chilena (Maureira, et al., 2014).

Inteligencia: Se utilizó el Test de matrices progresivas de Raven Escala General de Raven, Raven y Court (2003) que está compuesto por 60 problemas organizados en cinco series (A, B, C, D y E) de doce ítems cada uno. Con respecto a la validez y confiabilidad del test en Chile Ivanovic et al. mostraron niveles aceptables de validez concurrente y confiabilidad (Ivanovic, Forno, Durán, Hazbún, Castro \& Ivanovic, 2000).

\section{Procedimiento}

Las medidas antropométricas de 50 estudiantes de educación física se realizaron de forma individual en una sala acondicionada para ello. Las evaluaciones se realizaron a primera hora de la mañana, con pantalones cortos y parte superior de bikinis o top. El evaluador fue un profesor de educación física con vasta experiencia en mediciones antropométricas y con la certificación internacional de cineantropometría nivel 2 de la International Society for the Advancement of Kinanthropometry (ISAK). Las mediciones de la atención, memoria e inteligencia se realizaron a los 125 estudiantes en forma grupal en una sala de clases, con una duración aproximada de 70 minutos. Finalmente, en otra sesión se aplicaron las pruebas de cualidades físicas, en un gimnasio cerrado con piso de cemento. Antes de comenzar las evaluaciones se realizó un calentamiento dirigido de 10 minutos, luego se realizó la prueba de potencia abdominal, seguida de la prueba de potencia de brazos, potencia de piernas y potencia aeróbica. Entre cada prueba cada participante tuvo 20 minutos de descanso. Todos los participantes firmaron un consentimiento informado.

\section{Análisis de datos}

Se utilizó el programa estadístico SPSS 25.0 para Windows. Se aplicó estadística descriptiva con medias y desviaciones estándar, se analizó la distribución de las variables con pruebas KS de normalidad, tras lo cual se aplicó estadística inferencial con pruebas t para muestras independientes para comparar los valores de la composición corporal, cualidades físicas y pruebas cognitivas entre damas y varones. Finalmente, las correlaciones de Pearson permitieron estudiar la relación entre las variables estudiadas. Se consideró que un valor $\mathrm{p}<0,05$ era estadísticamente significativo.

\section{Resultados}

A continuación, en la tabla 1 se presentan las cuatro variables cognitivas (inteligencia, memoria visual, atención selectiva y sostenida) y las cuatro cualidades físicas (potencia abdominal, potencia de tren superior, potencia de tren inferior y capacidad aeróbica) del total de la muestra. Las pruebas t para muestras independientes no muestran diferencias en ninguna de las variables cognitivas según el sexo de la muestra ( $>00,05$ ), en tanto, como era de esperarse, en todas las cualidades físicas los varones presentan puntuaciones mayores $(p<0,001)$.

En la tabla 2 se describen y comparan las variables antropométricas según el sexo de la muestra. Los varones presentan mayores índices en gran parte de las variables estudiadas, sin embargo, no se observan diferencias en el IMC, diámetro iliocrestidio, perímetros tórax mesoesternal, cadera máxima, muslo máximo, muslo medial y pantorrilla, pliegue cutáneo abdominal, masa adiposa eíndice musculo-óseo.

En la tabla 3 se muestran las correlaciones de Pearson entre las variables cognitivas y diversas cualidades físicas-antropométricas, don- 
Tabla 1.

\begin{tabular}{|c|c|c|c|c|}
\hline Variables & $\begin{array}{c}\text { Media total } \\
(\mathrm{n}=125)\end{array}$ & $\begin{array}{c}\text { Media damas } \\
(\mathrm{n}=40)\end{array}$ & $\begin{array}{c}\text { Media varones } \\
(\mathrm{n}=85)\end{array}$ & Valor $p$ \\
\hline Inteligencia & $48,8 \pm 4,86$ & $48,6 \pm 4,83$ & $48,9 \pm 4,90$ & 0,748 \\
\hline Memoria visual & $6,5 \pm, 80$ & $6,6 \pm 1,76$ & $6,4 \pm 1,82$ & 0,695 \\
\hline Atención selectiva & $38,3 \pm 2,52$ & $38,8 \pm 3,16$ & $37,9 \pm 2,26$ & 0,728 \\
\hline Atención sostenida & $185,4 \pm 55,38$ & $182,5 \pm 72,02$ & $186,8 \pm 45,33$ & 0,693 \\
\hline Abdominal 60" & $47,4 \pm 9,55$ & $42,1 \pm 8,84$ & $49,9 \pm 8,86$ & 0,000 ** \\
\hline Flex-ext. de codos 30 & $25,4 \pm 7,18$ & $21,8 \pm 5,66$ & $27,2 \pm 7,22$ & $0,000^{* *}$ \\
\hline Salto largo a pies juntos & $1,90 \pm 0,30$ & $1,60 \pm, 19$ & $2,05 \pm 0,23$ & $0,000 * *$ \\
\hline Navetta & $7,3 \pm 2,18$ & $5,3 \#, 73$ & $8,3 \#, 64$ & $0,000 * *$ \\
\hline
\end{tabular}

Tabla 2

Medias, desviación estándar y pruebas $U$ de Mann-Whitney comparando las diversas variables antropométricas según el sexo de la muestra.

\begin{tabular}{|c|c|c|c|c|}
\hline & $\begin{array}{c}\text { Total } \\
(\mathrm{n}=50)\end{array}$ & $\begin{array}{l}\text { Damas } \\
(\mathrm{n}=16)\end{array}$ & $\begin{array}{l}\text { Varones } \\
(\mathrm{n}=34)\end{array}$ & Valor $p$ \\
\hline Peso & $67,3 \pm 0,98$ & $59,8 \pm 7,24$ & $70,7 \pm 0,75$ & $0,001^{* *}$ \\
\hline Talla & $169,4 \pm 9,05$ & $159,5 \pm 5,29$ & $174,1 \pm 6,26$ & $0,000^{* * *}$ \\
\hline Talla sentado & $90,1 \pm 3,83$ & $86,5 \pm 2,30$ & $91,8 \pm 3,19$ & $0,000^{* *}$ \\
\hline IMC & $23,4 \pm 2,99$ & $23,5 \pm 2,96$ & $23,3 \pm 3,05$ & 0,662 \\
\hline \multicolumn{5}{|l|}{ Diámetros (cm.) } \\
\hline Biacromial & $39,1 \pm 2,59$ & $36,2 \pm, 51$ & 40,5\#,71 & $0,000^{* *}$ \\
\hline Tórax transverso & $27,1 \pm 2,64$ & $25,3 \pm 2,34$ & $28,0 \pm 2,65$ & $0,000^{* *}$ \\
\hline Tórax antero-posterior & $20,4 \pm 2,93$ & $19,8 \pm 4,68$ & $20,7 \pm 1,60$ & $0,001^{* *}$ \\
\hline Iliocrestidio & $27,6 \pm, 69$ & $27,0 \pm, 38$ & $27,9 \pm 1,78$ & 0,098 \\
\hline Humeral & $6,7 \pm, 50$ & $6,1 \pm 0,28$ & $6,9 \pm 0,34$ & $0,000^{* *}$ \\
\hline Femoral & $9,6 \pm 0,61$ & $8,9 \pm 0,51$ & $9,9 \pm 0,43$ & $0,000^{* *}$ \\
\hline \multicolumn{5}{|l|}{ Perímetros (cm.) } \\
\hline Cabeza & $55,7 \pm 144$ & $55,0 \pm, 44$ & $55,9 \pm 1,36$ & $0,021^{*}$ \\
\hline Brazo relajado & $28,7 \pm 3,02$ & $27,3 \pm 2,25$ & $29,4 \pm 3,13$ & $0,031^{*}$ \\
\hline Brazo flexionado & $29,9 \pm 3,26$ & $27,4 \pm 2,20$ & $31,1 \pm 3,04$ & $0,000^{* *}$ \\
\hline Antebrazo máximo & $25,4 \pm 2,61$ & $23,3 \#, 17$ & $26,4 \pm 2,51$ & $0,000^{* *}$ \\
\hline Tórax mesoesternal & $91,5 \pm 6,21$ & $89,1 \pm 5,50$ & $92,7 \pm 6,27$ & 0,070 \\
\hline Cintura mínima & $74,7 \pm 8,73$ & $71,2 \pm 5,85$ & $76,4 \pm 9,41$ & $0,014^{*}$ \\
\hline Cadera máxima & $95,1 \pm 6,11$ & $95,8 \pm 6,56$ & $94,8 \pm 6,37$ & 0,473 \\
\hline Muslo máximo & $57,6 \pm 5,02$ & $58,7 \pm 4,62$ & $57,1 \pm 5,19$ & 0,244 \\
\hline Muslo medial & $53,0 \pm 4,78$ & $52,0 \pm 5,62$ & $53,5 \pm 4,85$ & 0,371 \\
\hline Pantorrilla & $35,6 \pm 2,58$ & $35,1 \pm, 26$ & $35,9 \pm 2,72$ & 0,252 \\
\hline \multicolumn{5}{|l|}{ Pliegues cutáneos (mm.) } \\
\hline Tríceps & $11,8 \pm 5,56$ & $16,8 \pm 4,47$ & $9,6 \pm 4,42$ & $0,000^{* *}$ \\
\hline Subescapular & $10,4 \pm 5,22$ & $12,0 \pm 5,44$ & $9,6 \pm 5,02$ & $0,036^{*}$ \\
\hline Suprailíaco & $10,7 \pm 5,97$ & $12,2 \pm 3,60$ & $10,1 \pm 6,75$ & $0,018^{*}$ \\
\hline Abdominal & $17.9 \pm 3,37$ & $18,5 \pm 4,91$ & $17,7 \pm 9,65$ & 0,261 \\
\hline Muslo & $16,5 \pm 3,07$ & $22,9 \pm 7,69$ & $13,4 \pm 6,32$ & $0,000^{* *}$ \\
\hline Pantorrilla & $10,4 \pm 6,10$ & $5,7 \pm, 42$ & $4,5 \pm 0,76$ & $0,000^{* *}$ \\
\hline Total pliegues & $103,1 \pm 29,29$ & $117,5 \pm 21,24$ & $96,3 \pm 30,31$ & $0,003^{* *}$ \\
\hline Masa adiposa (kl.) & $18,3 \pm 4,75$ & $18,7 \pm 3,01$ & $18,1 \pm 5,42$ & 0,236 \\
\hline Masa muscular (kl.) & $30,3 \pm 5,82$ & $25,4 \pm 3,42$ & $32,7 \pm 5,23$ & $0,000^{* *}$ \\
\hline Residual (kl.) & $7,3 \pm 1,83$ & $6,0 \pm 1,25$ & $7,8 \#, 79$ & $0,000^{* *}$ \\
\hline Masa ósea (kl.) & $7,8 \pm 1,28$ & $6,5 \pm 0,82$ & $8,4 \pm 0,97$ & $0,000^{* *}$ \\
\hline Piel (kl.) & $3,6 \pm 0,38$ & $3,3 \pm 0,20$ & $3,8 \pm 0,33$ & $0,000 * *$ \\
\hline Índice musculo-óseo & $3,9 \pm 0,46$ & $3,9 \pm 0,41$ & $3,9 \pm 0,48$ & 0,731 \\
\hline
\end{tabular}

Tabla 3.

Correlaciones de Pearson entre las variables cognitivas y las cualidades físicaantropométricas de la muestra.

\begin{tabular}{lcccc}
\hline antropométricas de la muestra. & & & & \\
\hline Abdominal 60" & Inteligencia & Memoria & A. selectiva & A. sostenida \\
Flex-ext. de codos 30" & NS & NS & NS & NS \\
Salto largo a pies juntos & †0,287* & NS & NS & p0,375* \\
Navetta & NS & NS & NS & p0,413* \\
\hline Estatura & NS & NS & NS & NS \\
Peso & NS & NS & NS & NS \\
IMC & NS & NS & NS & NS \\
Masa adiposa & NS & NS & NS & NS \\
Masa muscular & NS & NS & NS & NS \\
Residual & NS & NS & NS & NS \\
Masa ósea & NS & NS & NS & $\neq 0,285^{*}$ \\
& NS & NS & p0,535* & $\neq 0,310^{*}$ \\
Piel & NS & NS & NS & p0,602* \\
Índice musculo-óseo & NS & NS & NS & NS \\
\hline NS=no significativa; $\ddagger$ Total de la muestra; p damas; $†$ varones & &
\end{tabular}

de se observa relación entre los puntajes de inteligencia y atención sostenida con la potencia de tren superior e inferior y con los pliegues cutáneos del muslo y la pantorrilla; de las cuatro variables cognitivas con los diámetros biacromial, humeral y femoral; de la memoria y la atención sostenida con algunos perímetros y la atención con la masa residual y ósea.

\section{Discusión}

Los datos de la presente investigación arrojaron diferencias significativas en las cualidades físicas evaluadas entre varones y damas, siendo mayor las medias de los primeros. Estos resultados son similares a los encontrados por Durán, Valdés, Godoy y Herrera (2014), quienes evaluaron a 239 estudiantes de educación física con edades entre 18 y 31 años, donde los varones obtuvieron mayores puntuaciones en abdominales, flexo-extensiones de codo, salto largo a pies juntos y test Navetta. Esto se explica ya que la mayor cantidad de masa muscular de los universitarios varones incide positivamente en un mejor rendimiento en las pruebas de fuerza realizadas (López \& Fernández, 2006; Valdés,
Godoy, Herrera \& Durán, 2015), además las variables cinemáticas relacionadas con el desarrollo de maduración producen una mejor ejecución de la técnica de salto en varones, lo que ayuda a su mejor desempeño (Bermejo, López \& Palao, 2013).

Por otro lado, las mediciones de composición corporal mostraron diferencias en peso, talla sentado y en la mayoría de los diámetros medidos, siendo mayor en los varones, datos similares a otros estudios en poblaciones similares (Rodríguez, Berral, Almagià, Iturriaga \& Rodríguez, 2012; Vásquez, Castillo, Souza, Faundez \& Torrealba, 2018). En perímetros se encontró diferencias en brazo relajado, contraído y antebrazo, siendo mayor en hombres que en mujeres, esto básicamente tiene como explicación que los hombres poseen mayor masa muscular que las mujeres (Barbosa, Rodríguez, Hernández, de Valera, Hernández \& Herrera, 2007).

En pliegues cutáneos se encontró diferencia en la mayoría de las mediciones, datos muy similares a un estudio realizado por CossioBolaños, De Arruda, Moyano, Gañán, Pino y Lancho (2011) los cuales evaluaron a 125 estudiantes universitarios de 18 a 21 años, donde las mujeres obtuvieron medias superiores en todos los pliegues y menor masa magra, diferencias que pueden ser explicadas por los patrones de dimorfismo sexual, determinando a los varones con mayor masa ósea, muscular y residual, y las mujeres con superioridad del tejido adiposo.

Los análisis de correlaciones entre variables cognitivas y variables físicas muestran una relación baja de la inteligencia con la potencia del tren superior en varones con un $\mathrm{r}^{2}=0,08$. La memoria y la atención selectiva no presentan relación con ninguna cualidad física evaluada, pero la atención sostenida se correlaciona con la potencia de brazos y potencia de piernas en toda la muestra, con un $\mathrm{r}^{2}=0,14$ y r $^{2}=0,17$ respectivamente. Otros estudios anteriores han revelado una relación entre los niveles de control atencional y capacidad aeróbica en personas adultas, además de la atención y la velocidad en marcha en adultos mayores (Killane, et al., 2014; Pérez, et al., 2014; Wang, et al., 2015). También se ha constatado la relación entre la fuerza del tren inferior con la atención y memoria de trabajo (Scherder, Eggermont, Geuze, Vis \& Verkerke, 2010). Es interesante que ninguna prueba cognitiva hubiese presentado relación con la capacidad aeróbica, lo que suele ser lo usual en la literatura, al contrario, de estudios que muestren incidencia de la fuerza sobre atención, memoria o funciones ejecutivas (Maureira, 2018).

La inteligencia sólo presenta relación con una variable física evaluada, con una incidencia baja (cercana a 8\%), situación que ya había sido descrita por otros trabajos, donde no se encontró relación entre la práctica de ejercicio físico o una intervención con actividad física y la inteligencia (Lautenschlager, Cox, Flicker, Foster, van Bockxmeer, Xiao, et al., 2008; O'Callaghan, O'Callaghan, Williams, Bor y Najman, 2012). Esto encuentra sustento en las teorías jerárquicas de la inteligencia, que la presentan como una característica estable a través de la vida (Aravena \& Flores, 2017).

Los resultados muestran que la atención sostenida es la variable más influenciada por cualidades físicas y características antropométricas, situación que podría estar generada por las características de la evaluación, un proceso de diez minutos, donde el evaluado debe esta completamente enfocado en la resolución de la tarea, sin tiempos de descanso (a diferencia del test de memoria o inteligencia) lo que da cuenta de una necesidad continua de recursos cognitivos que podrían depender de mejores cualidades físicas que entreguen más recursos que ayuden a sostener el proceso.

Estos antecedentes sirven para intentar explicar las relaciones encontradas en el presente estudio, ya que parece ser que una mejor condición física se relaciona con mejor irrigación sanguínea, aumento de la vascularización, mayor consumo de glucosa a nivel cerebral, neurogénesis y sinaptogénesis (Cotman \& Berchtold, 2002; Voss, Heo, Prakash, Erickson, Alves, Chaddock, et al., 2013), siendo estos dos últimos procesos mediados por la acción de factores de crecimiento neuronal como el BDNF, FMRP, ARC, C-fos, etc. (Maureira, 2016). Todos estos procesos podrían asociar mejores cualidades físicas a mejores puntajes en pruebas que miden variables cognitivas.

En la presente investigación se analizaron cualidades físicas relacio- 
nadas con una mejor calidad de vida y variables cognitivas involucradas en el proceso aprendizaje cómo son la inteligencia, memoria, atención selectiva y sostenida, estando todas las pruebas validadas en esta población, con protocolos acorde al contexto nacional, constituyéndose como una fortaleza del presente estudio. Por otra parte, si bien la presente investigación trabajo con un alto porcentaje de estudiantes de primer año de la carrera de pedagogía en educación física de una Universidad de Santiago de Chile, sus resultados describen la realidad solo de la cohorte 2018, sin dar cuenta de la situación de los estudiantes en otros años de ingreso en la institución o la situación actual de estas variables en otro contexto regional o nacional, lo cual se constituye como una limitación del estudio.

\section{Conclusiones}

Se encontraron relaciones bajas entre la potencia de brazos e inteligencia en los varones, y relaciones medias entre la potencia de brazos y piernas con la atención sostenida en damas, en tanto, la memoria y la atención selectiva no presentaron relación con ninguna de las cualidades físicas evaluadas. Por otra parte, la atención selectiva se relacionó con la masa ósea en damas y la atención sostenida con la masa ósea y residual en damas y varones. Esto da cuenta de la existencia de relaciones entre variables cognitivas y cualidades físicas/antropométricas en la muestra evaluada, además de una influencia del sexo del estudiante sobre las relaciones establecidas.

Se recomienda realizar investigaciones cognitiva-físicaantropométrica con estudiantes de primer año de otras cohortes y en otras universidades, además de incluir otros años de carrera de pedagogía en educación física y evaluar la práctica de ejercicio físico, como un indicar que podría ser importante en la mediación de las variables aquí estudiadas.

\section{Referencias}

Agencia de la Calidad en Educación(2015). Estudio nacional de educación física. Recuperadodehttp://www.agenciaeducacion.cl/estudios/estudio-de-educacionfisica/

Aravena, C. \& Flores, E. (2017). Modelos jerárquicos y multifactoriales de la inteligencia II. En F. Maureira (Ed.) ¿Qué es la inteligencia? (pp. 41-50). Madrid: Bubok Publishing.

Barbosa, J., Rodríguez, N., Hernández, Y., de Valera, R., Hernández, H. \& Herrera, M.(2007). Masamuscular, fuerzamusculary otros componentes de funcionalidad en adultos mayores institucionalizados de la Gran Caracas-Venezuela. Nutrición Hospitalaria, 22(5), 578-583.

Benton L. (1981). Test de retención visual. Madrid: TEA

Bermejo, J., López, J. \& Palao, J. (2013). Diferencias de género en salto de altura según categorías de edad. Apunts. Educación Física y Deportes, 111(1), 62-69.

Braun, N., Gomes, F. \& Schütz, P. (2015). «The obesity paradox» in disease is the protective effect of obesity true? Swiss Medical Weekly, 145, w14265.

Cohen, S., Signorello, L., Cope, E., McLaughlin, J., Hargreaves, M., Zheng, W. et al. (2012). Obesity and all-cause mortality among black adults and white adults. American Journal of Epidemiology, 176(5), 431-442.

Cossio-Bolaños, M., De Arruda, M., Moyano, Á., Gañán, E., Pino, L. \& Lancho, J. (2011). Body composition of young university in relation to health. Nutrición Clínica y Dietética Hospitalaria, 31(3), 15-21.

Cotman, C. \& Berchtold, N. (2002). Exercise: a behavioral intervention to enhance brain health and plasticity. Trends Neuroscience, 25, 295-301.

Deckers, K., Van Boxtel, M., Verhey, F. \& Köhler, S. (2017). Obesity and cognitive decline in adults: effect of methodological choices and confounding by age in a longitudinal study. Journal of Nutrition Health and Aging, 21(5), 546-553.

Durán, S., Valdés, P., Godoy, A. \& Herrera, T. (2014). Hábitos alimentarios y condición física en estudiantes de pedagogía en educación física. Revista Chilena de Nutrición, 41(3), 251-259.

Eichen, D., Matheson, B., Liang, J., Strong, D., Rhee, K. \& Boutelle, K. (2018) Therelationship between executive functioning and weight loss and maintenance in children and parents participating in family-based treatment for childhood obesity. Behaviour Research and Theraphy, 105, 10-16.

Farooq, A., Gibson, A., Reilly, J. \& Gaoua, N. (2018). The association between obesity and cognitive function in otherwise healthy premenopausal arab women. Journal of Obesity, 1741962. doi: 10.1155/2018/1741962.

Gerard, K., Salicetti, A., Moncada, J. \& Solano, L. (2018). Mejora del equilibrio, atención y concentración después de un programa de entrenamiento exergame en la persona adulta mayor. Retos, 33, 102-105.

Goel, K., Lopez-Jiménez, F., De Schutter, A., Coutinho, T. \& Lavie, C. (2014). Obesity paradox in different populations: evidence and controversies. Future Cardiology, 10(1), 81-91.

Guillamón,A., García, E. \& Carrillo, P. (2019). Relación entre capacidad aeróbica y el nivel de atención en escolares de primaria. Retos, 35, 36-41.

Hirota, C., Watanabe, M., Sun, W., Tanimoto, Y., Kono, R., Takasaki, K., et al. (2010).Association between the Trail Making Test and physical performance in elderly Japanese. Geriatrics \& Gerontology International, 10(1), 40-47.

Huang, T., Tarp, J., Domazet, S., Thorsen, A., Froberg, K., Andersen, L., et al. (2015).Associations of adiposity and aerobic fitness with executive function and math performance in danish adolescents. Journal of Pediatric, 167(4), 810815.

Ivanovic, R., Forno, H., Durán, M., Hazbún, J., Castro, C. \& Ivanovic, D. (2000). Estudio de la capacidad intelectual (test de matrices progresivas de Raven) en escolares chilenos de 5 a 18 años I. Antecedentes generales, normas y recomendaciones. Revista de Psicología General y Aplicada, 53(1), 5-30.

Killane, I., Donoghue, O., Savva, G, Cronin, H., Kenny, R. \& Reilly, R. (2014). Relative association of processing speed, short-term memory and sustained attention with task on gait speed: a study of community-dwelling people 50 years and older. Journal of Gerontology A Biological Science \& Medical Science, 69(11), 1407-1414.

Lautenschlager, N., Cox, K., Flicker, L., Foster, J., van Bockxmeer, F., Xiao, J., et al. (2008). Effect of physical activity on cognitive function in older adults at risk for Alzheimer disease: a randomized trial. JAMA, 300, 1027-1037

León-Carrión J. (1995). Manual de neuropsicología humana. Madrid: siglo XXI. López, C. \&, Fernández, V.(2006). Fisiología del ejercicio. Madrid: Panamericana. Maureira, F. (2016). Plasticidad sináptica, BDNF y ejercicio físico. EmásF, Revista Digital de Educación Física, 7(40), 51-63.

Maureira, F.(2018). Principios deneuroeducación física. Madrid: Bubok Publishing.

Maureira, F., Trujillo, H. \& Flores, E. (2014). Propiedades psicométricas y datos normativos del test de atención Toulouse Piéron y del test de memoria visual de Benton forma $\mathrm{D}$ en estudiantes de educación física de Chile. Gaceta de Psiquiatría Universitaria, 10(2), 238-245.

Mullor, D., Gallego, J., Cangas, A., Aguilar-Parra, J., Valenzuela, L., Mateu, J., et al. (2017). Efectividad de un programa de actividad física en personas con trastorno mental grave. Revista Internacional de Medicina y Ciencias de la Actividad Física y el Deporte, 17(67), 507-521.

O’Callaghan, F., O'Callaghan, M., Williams, G., Bor, W. \& Najman, J. (2012). Physical activity and intelligence: a causal exploration. Journal of Physical Activity and Health, 9, 218-224

Pérez, L., Padilla, C., Parmentier, F. \& Andrés, P. (2014). The effects of chronic exercise on attentional networks. PLoS ONE, 9(7), e101478.

Raven, J.C., Raven J. \& Court J. (2003). Test de matrices progresivas: Escala General. Buenos Aires: Paidós.

Rodríguez, F., Berral, F., Almagià, A., Iturriaga, M. \& Rodríguez, F. (2012). Comparison of body composition and muscle mass body segment in students of physical education and sports of different disciplines. International Journal of Morphology,30(1), 7-14.

Scherder, E., Eggermont, L., Geuze, R., Vis, J. \& Verkerke, G. (2010). Quadriceps strength and executive functions in older women. American Journal of Physical Medicine \& Rehabilitation, 89(6), 458-463.

Skinner, J., Abel, W., McCoy, K. \& Wilkins, C. (2017). Exploring the «Obesity Paradox» as a correlate of cognitive and physical function in communitydwelling black and white older adults. Ethnicity \& Disease, 27(4), 387-394.

Valdés, P., Godoy, A., Herrera, T. \& Durán, S. (2015). Comparación en hábitos alimentarios y condición física entre estudiantes de educación física y otras carreras universitarias. Nutrición Hospitalaria, 32(2), 829-836.

Van der Niet,A., Smith, J., Scherder, E., Oosterlaan, J., Hartman, E. \& Visscher, C. (2015).Associations between daily physical activity and executive functioning in primary school-aged children. Journal of Science and Medicine in Sport, 18(6), 673-677.

Vásquez, J., Castillo, M., Souza, R., Faundez, C. \& Torrealba, A. (2018). Anthropometry, physical activity level and physical fitness in physical education students after four years in university. Nutrición Clínica y Dietética Hospitalaria, 38(1), 160-164.

Voss, M., Heo, S., Prakash, R., Erickson, K., Alves, H., Chaddock, L, et al. (2013). The influence of aerobic fittness on cerebral white matter integrity and cognitive function in older adults: results of a one-year exercise intervention. Human Brain Mapping, 34(11), 2972-2985.

Wang, C., Liang, W., Tseng, P., Muggleton, N., Juan, C. \& Tsai, C. (2015). The relation-ship between aerobic fitness and neural oscillations during visuospatial attention in young adults. Experimental Brain Research, 233(4), 10691078. 